\title{
Four Dimensional Realization of Two Dimensional Current Groups
}

\author{
Igor B. Frenkel, Boris A. Khesin \\ Department of Mathematics, Yale University, New Haven, CT 06520, USA
}

Received: 1 July 1995/Accepted: 17 July 1995

\begin{abstract}
The quotient realization of the central extensions of the current groups over Riemann surfaces is achieved by means of the Leray residue theory. This approach replaces de Rham cohomology in the classical WZNW construction for affine Lie groups.
\end{abstract}

\section{Introduction}

In the past decade the theory of affine Lie algebras and of the corresponding groups has become an established field in mathematics and theoretical physics with diverse connections to other areas in both disciplines. The geometric aspect of the theory was significantly advanced with an explicit realization of the affine Lie groups as quotients of central extensions of the current groups over a two-dimensional disk [W1, PS, M]. This construction is achieved by a striking combination of the basic facts from the theory of simple Lie groups and of de Rham cohomology. The realization of affine Lie groups is also directly related to the geometric approach to representation theory and a class of two-dimensional conformal field theories known as the WZNW model.

There were numerous attempts to generalize affine Lie algebras and groups to higher dimensions. However, corresponding generalizations of representation theory as well as of conformal field theory always encountered substantial difficulties. In $[\mathrm{EF}]$ there was introduced a class of central extensions of two dimensional current algebras and groups on a Riemann surface $\Sigma$ (endowed with a complex structure) with values in a complex simple Lie group $G$ that can be viewed as a natural generalization of the one dimensional counterpart. In particular, it was shown in [EF, EK] that the orbits in the coadjoint representation for these groups have a structure similar to those for loop groups. This fact strongly indicates that there exists a rich representation theory that generalizes the classical case of affine Lie groups. It was also shown that the central extension $\hat{G}^{\Sigma}$ of the current group $G^{\Sigma}=C^{\infty}(\Sigma, G)$ topologically is a nontrivial fibre bundle with a fibre isomorphic to the Jacobian variety of $\Sigma[\mathrm{EF}]$. 
The analogy between loop groups (or affine Lie groups) and double loop groups (or central extensions of current groups on Riemann surfaces) extends a long series of parallel constructions based respectively on real and complex numbers. In fact, the central extension $\hat{G}^{\Sigma}$ is intrinsically complex, and it does not admit a natural real form. On the other hand, the original definition of $\hat{G}^{\Sigma}$ in $[\mathrm{EF}]$ is based on a real construction of [PS]. The main motivation for this work was an attempt to find a direct complex realization of this central extension $\hat{G}^{\Sigma}$ that could serve as a starting point for geometric construction of representations. It turns out that there exists a quotient construction of $\hat{G}^{\Sigma}$ analogous in every step to that for affine Lie groups. In particular, one has to increase by one the complex dimension of the quotient construction rather than the real dimension as in the case of affine Lie groups.

The key element of the realization is embedding of the complex curve $\Sigma$ to a complex surface $N$ (i.e. to a manifold of real dimension 4). To obtain a counterpart of the topological 3-dimensional term of the classical construction we further embed $N$ into a complex three-dimensional manifold $M$ (of real dimension 6) and represent $\Sigma$ as a transverse intersection of two complex surfaces $N$ and $N^{\prime}$. The corresponding central extension is constructed with the help of the space of meromorphic forms on $M$ with poles along $N$ and $N^{\prime}$. The Leray residue theory in complex analysis now replaces the de Rham cohomology. Our main result is an explicit "formula" for the central extension of $\hat{G}^{\Sigma}$ developing in a rather unexpected way the construction of affine Lie groups in [M].

The case of punctured Riemann surfaces fits naturally the meromorphic setting of the paper: the corresponding meromorphic forms have poles along additional curves in $N$ and surfaces in $M$ which meet $\Sigma$ at the set of punctures.

Some ingredients of our construction have certain similarity to the four-dimensional analogues of conformal field theories [NS, LMNS], which were important stimulus for our work. However, there are fundamental differences between the two theories (see Sects. 4.1 and 5.3 for discussion). We believe that our realization brings us one step closer towards representation theory of two dimensionl current groups and their relation to four-dimensional analogues of conformal field theories.

We tried to make this paper essentially self-contained adding some basic definitions and results. In Sect. 1 we recall the construction of affine Lie algebras and the corresponding groups, including the direct quotient construction. In Sect. 2, we review the results from $[\mathrm{EF}]$ on central extension of current algebras and current groups in two dimensions and generalize them to currents over Riemann surfaces with punctures. After Sect. 3 outlining the preliminaries on the Leray theory, we present four dimensional realization of two dimensional current groups in Sect. 4. We consider the case of punctured Riemann surfaces in Sect. 5.

\section{Affine Lie Algebras and Groups}

1.1. Affine Lie algebras. Let $G$ be a simply connected simple compact Lie group, and let $\mathscr{G}$ be its Lie algebra. Denote by $\operatorname{Tr}(\cdot)$ the trace in $\mathscr{G}$ for the adjoint representation of $G$. In particular, $\operatorname{Tr}(X Y), X, Y \in \mathscr{G}$ is the Killing form on $\mathscr{G}$. We normalize our form by the following condition: if $h_{\alpha}$ is a coroot corresponding to a longest root $\alpha$, then $\operatorname{tr}(X Y):=2 \operatorname{Tr}(X Y) / \operatorname{Tr}\left(h_{\alpha}^{2}\right)$ so that $\operatorname{tr}\left(h_{\alpha}^{2}\right)=2$, see [PS]. 
Definition 1.1. The loop algebra $\mathscr{G} S^{1}$ is the set $C^{\infty}\left(S^{1}, \mathscr{G}\right)$ of all $\mathscr{G}$-valued smooth functions on the circle, endowed with the pointwise commutator. The (untwisted) affine Lie algebra $\hat{\mathscr{G}}^{S^{1}}$ is the one-dimensional nontrivial central extension of the Lie algebra $\mathscr{G}^{S^{1}}$ :

$$
0 \rightarrow \mathbb{R} \rightarrow \hat{\mathscr{G}}^{S^{1}} \rightarrow \mathscr{G}^{S^{1}} \rightarrow 0 .
$$

Explicitly, $\mathscr{G}^{1}$ is a linear space of all pairs $\left\{(X, a) \mid X \in C^{\infty}\left(S^{1}, \mathscr{G}\right), a \in \mathbb{R}\right\}$ with the commutator given by the relation

$$
[(X(t), a),(Y(t), b)]=((X Y-Y X)(t), c(X, Y)),
$$

where $c(X, Y)$ is the Maurer-Cartan $\mathbb{R}$-valued 2-cocycle

$$
c(X, Y)=\frac{1}{2 \pi} \int_{S^{1}} \operatorname{tr}(X(t) d Y(t)) .
$$

1.2. Affine Lie groups. Let $G^{S^{1}}=C^{\infty}\left(S^{1}, G\right)$ be the loop group with the pointwise product corresponding to the Lie algebra $\mathscr{G}^{1}$.

Theorem 1.2 ([PS]). There exists a central extension $\hat{G}^{S^{1}}$ of the group $G^{S^{1}}$ by means of the circle $S^{1}$

$$
1 \rightarrow S^{1} \rightarrow \hat{G}^{S^{1}} \rightarrow G^{S^{1}} \rightarrow 1,
$$

with the Lie algebra $\hat{\mathscr{G}}^{S^{1}}$. Moreover, the universal extension $\hat{G}^{S^{1}}$ in the category of Lie groups is simply connected, and it is singled out by this property among all extensions of $G^{S^{1}}$ by means of $S^{1}$.

However, the explicit construction of the corresponding affine Lie groups is not as straightforward as that of the Lie algebras, since the group central extension is topologically nontrivial.

1.3. Affine Lie groups as quotients. The following explicit realization of $\hat{G}^{S^{1}}$ as a quotient group was obtained in $[\mathrm{M}]$ and is implicitly contained in the WZNW model [W1], and in the more general topological approach of [PS]. We are splitting the construction into several steps for comparison with the higher-dimensional counterpart.

The group $G^{S^{1}}$ can be regarded as the quotient $G^{D} / G_{S}^{D}$, where $G^{D}:=C^{\infty}(D, G)$ is the group of currents on the 2-disk $D$ and $G_{S}^{D}$ is the subgroup of $G^{D}$ which consists of the currents equal to the identity $i d \in G$ on the boundary $\partial D=S^{1}$ :

$$
G_{S}^{D}:=\left\{g \in G^{D}|g|_{S^{1}=\partial D}=i d\right\} .
$$

Let $\hat{G}^{D}$ be the central extension of the group $G^{D}$ by a circle $S^{1}=\mathbb{R} / 2 \pi \mathbb{Z}$ defined by the 2-cocycle

$$
\gamma\left(g_{1}, g_{2}\right)=\frac{1}{4 \pi} \int_{D} \operatorname{tr}\left(g_{1}^{-1} d g_{1} \wedge d g_{2} g_{2}^{-1}\right) \bmod 2 \pi,
$$

where $g_{1}, g_{2} \in G^{D}$ are two group currents. 
Note, that the infinitesimal version of this cocycle for the Lie algebra $\hat{\mathscr{G}}^{D} \ni X, Y$ is

$$
c(X, Y)=\frac{1}{2 \pi} \int_{D} \operatorname{tr}(d X \wedge d Y)=\frac{1}{2 \pi} \int_{S^{1}} \operatorname{tr}(X d Y),
$$

where the latter expression (being the standard form of the cocycle of affine Lie algebras on $S^{1}$ ) is obtained by application of the Stokes formula to $\partial D=S^{1}$.

Theorem $1.3([\mathrm{M}])$. There is an embedding of the gauge group $G_{S}^{D}$ into $\hat{G}^{D}$ as a normal subgroup such that the quotient $\hat{G}^{D} / G_{S}^{D}$ is isomorphic to the extended loop group $\hat{G}^{S^{1}}$.

1.4. Construction of the embedding. The embedding mentioned is constructed as follows. Consider a three-dimensional ball $B$ with the boundary $S^{2}$ presented as a union of two disks $D$ and $D^{\prime}$ which intersect along a common circle $\partial D=\partial D^{\prime}=S^{1}$.

Given a $G$-valued map $g \in G_{S}^{D}$ we extend it by identity to the sphere $S^{2}$ and consider an arbitrary extension $\bar{g} \in C^{\infty}(B, G)$ of it to a map of the 3-dimensional ball $B$ "filling in" $S^{2}$ to the group $G$.

Proposition 1.4. The number

$$
\lambda(g)=-\frac{1}{12 \pi} \int_{B} \operatorname{tr}\left(\bar{g}^{-1} d \bar{g}\right)^{3}
$$

is well-defined modulo $2 \pi$ in spite of the ambiguity of the extension $\bar{g}$ for the map $g$.

Indeed, any two such extensions $\bar{g}$ and $\overline{\bar{g}}$ are related by a factor $h \in C^{\infty}(B, G)$ such that $\left.h\right|_{\partial B=S^{2}}=i d$. Thus it represents a homotopy class in $\pi_{3}(G) \simeq \mathbb{Z}$ and is determined by the integral $\frac{1}{24 \pi^{2}} \int_{S^{3}} \operatorname{Tr}\left(h^{-1} d h\right)^{3}$.

Now the desired embedding is $G_{S}^{D} \ni g \rightarrow(g, \lambda(g) \bmod 2 \pi) \in \hat{G}^{D}$ thanks to the relation

$$
\lambda\left(g_{1} g_{2}\right)-\lambda\left(g_{1}\right)-\lambda\left(g_{2}\right)=\gamma\left(g_{1}, g_{2}\right) .
$$

One can check that the image of $G_{S}^{D}$ is a normal subgroup in $\hat{G}^{D}$, and we will do it in a more general setting in Sect. 4.

One can define the cocycle $\gamma\left(g_{1}, g_{2}\right)$ modulo $2 \pi k$ for any real $k$. However, the embedding $G_{S}^{D} \rightarrow \hat{G}^{D}$ exists only when $k \in \mathbb{Z}$. Thus the topology of $G$ imposes restrictions on possible central extensions of $G^{D}$ corresponding to the Lie algebra $\hat{\mathscr{G}}^{S^{1}}$. We call $k \in \mathbb{Z}$ the level of the central extension, and the basic extension is the one associated to the level 1 . This notion corresponds to representations of level $k$ of the affine Lie algebra $\hat{\mathscr{G}}^{S^{1}}$.

We conclude this section with a note that all the above constructions remain unchanged if we choose $G$ to be a complex simply connected simple Lie group, $\mathscr{G}$ the corresponding complex Lie algebra and consider central extensions with values in $\mathbb{C}$. It turns out that the constructions of the next sections are intrinsically complex and do not admit a straightforward real form. 


\section{The Lie Algebra and Group of Currents over a Riemann Surface}

2.1. The current Lie algebra over a Riemann surface. Let $G$ be a simply connected simple complex Lie group, and $\Sigma$ a nonsingular oriented two-dimensional closed surface of genus g. Define $G^{\Sigma}=C^{\infty}(\Sigma, G)$ to be the group of all smooth mappings from $\Sigma$ to $G$. Its Lie algebra consists of all smooth $\mathscr{G}$-valued functions on $\Sigma$, and it is denoted by $\mathscr{G}^{\Sigma}$.

Fix a complex structure on the surface $\Sigma$. Denote by $\mathscr{H}^{1}(\Sigma)$ the space of holomorphic differentials on $\Sigma$. It has the complex dimension $\mathbf{g}$.

Definition 2.1. The central extension $\hat{\mathscr{G}}^{\Sigma}$ of the current algebra $\mathscr{G}^{\Sigma}$ with the center $\mathscr{H}^{1}(\Sigma)^{*}$ is defined by means of the $\mathscr{H}^{1}(\Sigma)^{*}$-valued 2-cocycle $C(X, Y), X, Y \in \mathscr{G}^{\Sigma}$ such that

$$
\langle C(X, Y), \theta\rangle=\int_{\Sigma} \operatorname{tr}(X d Y) \wedge \theta
$$

for any holomorphic differential $\theta \in \mathscr{H}^{1}(\Sigma)$.

2.2. The case of punctured surfaces. The construction above can be generalized to the case of a punctured Riemann surface. The form $\theta$ admits poles on a finite set $\Pi=\left\{p_{1}, \ldots, p_{r}\right\}$ of $\Sigma$. We will only consider the case of simple poles. Denote by $\mathscr{G}_{\Pi}^{\Sigma}$ a subalgebra of $\mathscr{G}^{\Sigma}$ which consists of $\mathscr{G}$-valued functions on $\Sigma$ assuming zero values at each point of $\Pi$. Let $\mathscr{H}^{1}(\Sigma \backslash \Pi)$ denote the space of meromorphic differentials on $\Sigma$ with possible simple poles at the points of $\Pi\left(\operatorname{dim}_{\mathbb{C}} \mathscr{H}^{1}(\Sigma \backslash \Pi)=\right.$ $\mathbf{g}+r-1)$.

Proposition 2.2. The formula (2.1) for $\theta \in \mathscr{H}^{1}(\Sigma \backslash \Pi)$ gives rise to a 2-cocycle on $\mathscr{G}_{\Pi}^{\Sigma}$ with values in $\mathscr{H}^{1}(\Sigma \backslash \Pi)^{*}$.

Proof. Let $U_{\varepsilon}\left(p_{i}\right)$ be a circle of radius $\varepsilon$ around a point $p_{i} \in \Pi$ in a local coordinate $z_{i}$ and let $\Sigma_{\varepsilon}:=\Sigma \backslash\left(\bigcup_{l=1}^{n} U_{\varepsilon}\left(p_{i}\right)\right)$. Then for any function $f \in C^{\infty}(\Sigma, \mathbb{C})$ we have

$$
\int_{\Sigma} d f \wedge \theta:=\lim _{\varepsilon \rightarrow 0} \int_{\Sigma_{\varepsilon}} d f \wedge \theta=-\lim _{\varepsilon \rightarrow 0} \sum_{i=1}^{n} \oint_{\left|z_{l}\right|=\varepsilon} f(z) \theta(z)=-2 \pi i \sum_{j=1}^{n} f\left(p_{j}\right) \operatorname{res} \theta\left(p_{j}\right)
$$

by the Green theorem. This implies skew-symmetry of the form $C(X, Y)$ for currents $X, Y$ vanishing on $\Pi$ :

$$
\langle C(X, Y)+C(Y, X), \theta\rangle=\int_{\Sigma} d(\operatorname{tr}(X Y)) \wedge \theta=-2 \pi i \sum_{j=1}^{n} \operatorname{tr}\left(X Y\left(p_{j}\right)\right) \operatorname{res} \theta\left(p_{j}\right)=0 .
$$

Similarly one verifies the cocycle identity $\sum_{\{X, Y, Z\}} C([X, Y], Z)=0$.

The Green theorem above has a far reaching generalization based on the Leray theory of higher dimensional residues. This simple yet fundamental fact underlies our consideration.

Note, that Proposition 2.2 holds for a more general (than $\mathscr{G}_{\Pi}^{\Sigma}$ ) subalgebra of $\mathscr{G}^{\Sigma}$ consisting of $\mathscr{G}$-valued functions on $\Sigma$ which at each point $p_{j} \in \Pi$ assume values at a certain nilpotent subalgebra (depending on $j$ ). 


\subsection{Central extensions of the current group.}

Theorem 2.3 ([EF]). There exists a central extension $\hat{G}^{\Sigma}$ of the current group $G^{\Sigma}$ by means of the Jacobian variety $\mathscr{J}$ of the Riemann surface $\Sigma$, such that the Lie algebra of the extensions is $\hat{\mathscr{G}}^{\Sigma}$.

This theorem is a counterpart of Theorem 1.2 (due to [PS]), and the group can be constructed by the procedure described in [PS, Chapter 4], see [EF]. Namely, one defines the left invariant holomorphic 2 -form $\tilde{C}$ on $G^{\Sigma}$ equal to $C(\cdot, \cdot)$ on the tangent space at the identity $i d \in G^{\Sigma}$. The integrals of this form over integer 2-cycles in $G^{\Sigma}$ fill the lattice $L=H_{1}(\Sigma, \mathbb{Z})$ in $\mathscr{H}^{1}(\Sigma)^{*}$. Therefore, there exists a holomorphic principal bundle over $G^{\Sigma}$ with fiber $\mathscr{J}=\mathscr{H}^{1}(\Sigma)^{*} / L$ being the Jacobian of the surface $\Sigma$, and with a holomorphic connection whose curvature form is $2 \pi \tilde{C}$. The group $\hat{G}^{\Sigma}$ is the group of all transformations of the constructed bundle that preserve the connection and project to left translations on the group $G^{\Sigma}$. It is the desired central extension of $G^{\Sigma}$ by $\mathscr{J}$.

A straightforward modification of this argument leads to the following

Theorem 2.3'. The current group $G_{\Pi}^{\Sigma}$ on the Riemann surface $\Sigma$ punctured at $\Pi$ can be extended by means of the Jacobian $\mathscr{J}^{\prime}=\mathscr{H}^{1}(\Sigma \backslash \Pi)^{*} / L^{\prime}$, where the lattice $L^{\prime}$ is $L^{\prime}=H_{1}(\Sigma \backslash \Pi, \mathbb{Z})$ (and for $\Sigma$ of genus $\mathbf{g}$ it is spanned by $2 \mathbf{g}$ independent 1-cycles on $\Sigma$ and by contours around each but one removed point $p_{i}$ ).

\section{Preliminaries on the Leray Theory}

3.1. The Leray residue. Let $M$ be an $n$-dimensional compact complex manifold, and $N \subset M$ a nonsingular complex hypersurface (such that for every point $p_{0} \in N$ the submanifold $N$ is defined in a neighborhood $U\left(p_{0}\right)$ as a zero level of some holomorphic function $\psi_{p_{0}}$ with nonzero gradient).

Let $\omega$ be a closed $C^{\infty}$-smooth $k$-form on $M \backslash N$ which has a pole of the first order on $N$. The latter means that the product $\psi_{p_{0}} \cdot \omega$ can be extended to a $C^{\infty}$. form in $U\left(p_{0}\right)$. Throughout the paper we assume that all poles are of the first order unless the opposite is explicitly stated.

Theorem-Definition 3.1. In a neighborhood $U\left(p_{0}\right)$ of an arbitrary point $p_{0} \in N$ the closed $k$-form $\omega$ can be decomposed into the sum

$$
\omega=\frac{d \psi}{\psi} \wedge \theta+\alpha
$$

outside of $N$, where the forms $\theta$ and $\alpha$ are smooth in $U\left(p_{0}\right)$. The restriction $\left.\theta\right|_{N}$ is a well-defined (i.e. independent of the choice of $\psi)$ closed $(k-1)$-form. The form-residue res $\omega$ of the form $\omega$ is the closed $(k-1)$-form on $N$ such that in any neighborhood $U\left(p_{0}\right)$ of an arbitrary point $p_{0} \in N$ it coincides with the form $\left.\theta\right|_{N}$ of the decomposition (3.1): res $\omega=\left.\theta\right|_{N}$. If $\omega$ is holomorphic on $M \backslash N$, then res $\omega$ is holomorphic on $N$.

Note that a cohomology class of res $\omega$ depends only on a class of $\omega$. Thus we get a map

$$
\operatorname{Res}: H^{k}(M \backslash N) \rightarrow H^{k-1}(N) \text {. }
$$


3.2. The coboundary operator. Define now the coboundary Leray operator

$$
\delta: H_{k-1}(N) \rightarrow H_{k}(M \backslash N)
$$

between the homology groups (of chains with compact support). This operator associates to every point $p_{0} \in N$ a topological circumference $\delta p_{0} \subset M \backslash N$ such that the following three conditions hold.

Assume that in local coordinates $\left(z_{1}, \ldots, z_{n}\right)$ in a neighborhood $U\left(p_{0}\right)$ the surface $N \cap U\left(p_{0}\right)$ is defined by the constraint $z_{n}=0$. Then 1$)$ the circle $\delta p_{0} \subset U\left(p_{0}\right)$ is determined by the equation $\left|z_{n}\right|=1$; 2) the set $\bigcup_{p \in N} \delta p$ is continuous; and 3) $\delta p^{\prime} \cap$ $\delta p^{\prime \prime}=\emptyset$, whereas $p^{\prime} \neq p^{\prime \prime}$.

Now any $(k-1)$-cycle $\sigma$ on $N$ gives rise to a $k$-cycle in $M \backslash N$. Moreover, the $\delta$-operator commutes with the de Rham boundary operator $\partial$, therefore, we have an operator between the corresponding homology groups

$$
\delta: H_{k-1}(N) \rightarrow H_{k}(M \backslash N) .
$$

Theorem 3.2 ([L]). Let $\sigma \subset N$ be a $(k-1)$-dimensional cycle in $N$ and let $\omega \in$ $C^{\infty}(M \backslash N)$ be a closed $k$-form with a polar set $N$ of order 1 . Then

$$
\int_{\delta \sigma} \omega=2 \pi i \int_{\sigma} \operatorname{res} \omega .
$$

The theory above admits a natural generalization. Let $N_{1}, \ldots, N_{r}$ be complex submanifolds of $M$ of codimension 1 in a general position. Let $\omega$ be a smooth closed $k$-form on $M \backslash N$ (where $N:=N_{1} \cup \cdots \cup N_{r}$ ) which has a pole of the first order on all the submanifolds (and respectively, the pole is of order higher than 1 on their mutual intersections).

Then one can define the residue form res $\omega$ as above in generic points of $N_{1}$, i.e. on $N_{1} \backslash\left(N_{1} \cap\left(N_{2} \cup \cdots \cup N_{r}\right)\right)$. The residue res $\omega$ will have poles on the submanifolds $N_{1} \cap N_{2}, \ldots, N_{1} \cap N_{r}$, etc. The corresponding operations Res and $\delta$ on cohomology and homology of the above spaces are well defined and are related by Theorem 3.2.

One can go further and compose the Leray operations. Say, for two submanifolds $N_{1}, N_{2}$ in $M$ one has the mappings

$$
\begin{gathered}
\operatorname{Res}^{2}: H^{k}\left(M \backslash\left(N_{1} \cup N_{2}\right)\right) \rightarrow H^{k-2}\left(N_{1} \cap N_{2}\right) \\
\delta^{2}: H_{k-2}\left(N_{1} \cap N_{2}\right) \rightarrow H_{k}\left(M \backslash\left(N_{1} \cup N_{2}\right)\right) .
\end{gathered}
$$

Theorem 3.2 now reads that for any $(k-2)$-cycle $\sigma$ representing an element of $H_{k-2}\left(N_{1} \cap N_{2}\right)$ and for any closed $C^{\infty} k$-form $\omega$ whose cohomology class is in $H^{k}\left(M \backslash\left(N_{1} \cup N_{2}\right)\right)$ we have

$$
\int_{\delta^{2} \sigma} \omega=(2 \pi i)^{2} \int_{\sigma} \operatorname{res}^{2} \omega .
$$

3.3. The Leray-Stokes Theorem. The following statement relating de Rham and Leray theories is a cornerstone of the further constructions.

Let $M$ be a complex compact manifold of complex dimension $n$, and $N$ a complex hypersurface. 
Theorem 3.4 (cf. e.g. [GS]). Assume that a $2 n$-form $\mu=d \alpha \wedge \rho$ on $M \backslash N$ is a wedge product of an exact smooth $n$-form $d \alpha$ with $\alpha \in \Omega^{n-1}(M)$ and a meromorphic n-form $\rho$ on $M$ with a polar set $N$ of order 1 . Then the form $\mu$ is an integrable form on $M$, and

$$
\int_{M} d \alpha \wedge \rho=-2 \pi i \int_{N} \alpha \wedge \operatorname{res} \rho .
$$

Proof. To show that $\mu \in L^{1}(M)$ recall that in a neighborhood of any point $p_{0} \in N$ (where in a coordinate system $\left(z_{1}, \ldots, z_{n}\right)$ the polar submanifold $N$ is defined by the condition $z_{n}=0$ ) the form $\mu$ grows at a rate $\frac{1}{\left|z_{n}\right|}$ as $z_{n} \rightarrow 0$. On the other hand, the (real) codimension of $N$ equals 2, that makes all the improper integrals converge.

Split $M$ into two parts by a submanifold $\delta_{\varepsilon} N$ (where $\delta$ is the Leray operator, sending a point $p_{0} \in N$ into the circumference $\left.\left|z_{n}\right|=\varepsilon\right): M=U_{\varepsilon}(N) \cup \overline{U_{\varepsilon}(N)}$ so that $U_{\varepsilon}(N)$ is an $\varepsilon$-neighborhood of $N$. Then by definition of an improper integral for an $L^{1}$-form we obtain

$$
\int_{M} \mu:=\lim _{\varepsilon \rightarrow 0} \int \frac{\int_{\bar{\varepsilon}}(N)}{\left.U^{(}\right)} d \alpha \wedge \rho=-\lim _{\varepsilon \rightarrow 0} \int_{\delta_{\varepsilon} N} \alpha \wedge \rho=-\lim _{\varepsilon \rightarrow 0} \int_{N} \oint_{\left|z_{n}\right|=\varepsilon} \alpha \wedge \rho,
$$

where the form $\mu$ is smooth in $\overline{U_{\varepsilon}(N)}$ and the Stokes formula reduces the integral to that over $\delta_{\varepsilon} N=\partial U_{\varepsilon}(N)=-\partial \overline{U_{\varepsilon}(N)}$.

In a neighborhood of every $p_{o} \in N$ the form $\rho$ decomposes as $\rho=\frac{d z_{n}}{z_{n}} \wedge \theta+\beta$ with holomorphic $\theta$ and $\beta$. Hence

$$
\int_{M} \mu=-\int_{N} \alpha \wedge\left(\lim _{\varepsilon \rightarrow 0} \oint_{\left|z_{n}\right|=\varepsilon}\left(\frac{d z_{n}}{z_{n}} \wedge \theta+\beta\right)\right) .
$$

As $\varepsilon \rightarrow 0$, the contribution of $\alpha \wedge \beta$ vanishes, while Leray Theorem 3.2 (or the classical Cauchy theorem) reduces the integral of the first term to the corresponding residue: $\int_{M} \mu=-2 \pi i \int_{N} \alpha \wedge \operatorname{res} \rho$.

It is clear from the proof that Theorem 3.4 remains true if $\rho$ is allowed to have poles of the first order along a union $N_{1} \cup \cdots \cup N_{r}$ of manifolds of codimension 1 .

3.4. Meromorphic setting of the Leray theory. It turns out that the construction of central extensions of the current groups uses naturally the language of meromorphic differential forms. We are grateful to A. Todorov for detailed explanations of the results on Leray cycles. We formulate the theorems of this section in the minimal generality required below. For more general setting we direct the reader to references [T, GS, Ch].

Let $\mathscr{H}^{k}(M)$ be the space of holomorphic $k$-forms on a manifold $M$. For a hypersurface $N \subset M$ we denote by $\mathscr{H}^{k}(M \backslash N)$ the space of meromorphic $k$-forms with poles of the first order on $N$. Note that for $k=n=\operatorname{dim}_{\mathbb{C}} M$ the holomorphic forms are automatically closed. Moreover, if $M$ is Kähler, they are harmonic and therefore the space $\mathscr{H}^{n}(M)$ is isomorphic to the Hodge space $H^{n, 0}(M, \mathbb{C})$.

Theorem 3.5 (e.g. [Ch, Chapter 15]). For a hypersurface $N \subset M$ in a complex $n$-dimensional manifold $M$ with vanishing Hodge dimension $h^{n, 1}(M)=0$ one has 
the following exact sequence:

$$
0 \rightarrow \mathscr{H}^{n}(M) \rightarrow \mathscr{H}^{n}(M \backslash N) \rightarrow \mathscr{H}^{n-1}(N) \rightarrow 0,
$$

where the maps are inclusion and taking the residue respectively.

Proof sketch. Present an arbitrary holomorphic $(n-1)$-form $\theta \in \mathscr{H}^{n-1}(N)$ as the distribution form $\theta \wedge \xi_{N}$ on $M$, where $\xi_{N}$ is the 2-form with support on $N$ (and Poincare dual to $N$ itself). Then $\bar{\partial}\left(\theta \wedge \xi_{N}\right)=0$, because $\theta$ is holomorphic and $N$ is a complex surface. Since $h^{n, 1}(M)=0$, the $\bar{\partial}$-closed form $\theta \wedge \xi_{N}$ is $\bar{\partial}$-exact: $\theta \wedge \xi_{N}=$ $\bar{\partial} \omega$ for some $\omega \in \mathscr{H}^{n}(M \backslash N)$. The latter means that res $\omega=\theta$.

Remark. This proof as well as most of what follows admits an alternative algebrogeometric description. For instance, start with a short exact sequence of sheaves of holomorphic functions:

$$
0 \rightarrow \mathcal{O}_{M}(-N) \rightarrow \mathcal{O}_{M} \rightarrow \mathcal{O}_{N} \rightarrow 0
$$

where $\mathcal{O}_{M}(-N)$ is the sheaf of functions vanishing on $N$. Then tensor multiplication by $\mathcal{O}_{M}(N)$, the (inverse) sheaf of functions on $M$ associated to a divisor $N$ gives

$$
\left.0 \rightarrow \mathcal{O}_{M} \rightarrow \mathcal{O}_{M}(N) \rightarrow \mathcal{O}_{M}(N)\right|_{N} \rightarrow 0 \text {. }
$$

Now the tensor product of this sequence with sheaf of holomorphic $n$-forms $\Omega_{M}^{n}$ on $M$ yields

$$
\left.0 \rightarrow \Omega_{M}^{n} \rightarrow \Omega_{M}^{n}(N) \rightarrow \Omega_{M}^{n}(N)\right|_{N} \rightarrow 0 \text {. }
$$

Notice that $\left.\Omega_{M}^{n}(N)\right|_{N} \simeq \Omega_{N}^{n-1}$ due to Theorem-Definition 3.1. It allows one to write the corresponding long exact sequence

$$
0 \rightarrow H^{0}\left(\Omega_{M}^{n}\right) \rightarrow H^{0}\left(\Omega_{M}^{n}(N)\right) \rightarrow H^{0}\left(\Omega_{N}^{n-1}\right) \rightarrow H^{1}\left(\Omega_{M}^{n}\right),
$$

the last term of which vanishes by virtue of the assumption. This is the desired sequence (3.2) modulo notations.

The main application of the Leray theory below is the case of a 3-dimensional complex manifold $M$ and a pair of 2-dimensional complex submanifolds $N_{1}, N_{2} \subset M$ whose intersection $N_{1} \cap N_{2}$ is a complex curve $\Sigma$. Now let us fix a holomorphic 1 -form $\theta$ on $\Sigma=N_{1} \cap N_{2}$.

Corollary 3.6. The (complex) dimension of the space of all meromorphic 3-forms $\eta \in \mathscr{H}^{3}\left(M \backslash\left(N_{1} \cup N_{2}\right)\right)$ is equal to $\operatorname{dim} \mathscr{H}^{1}(\Sigma)+\operatorname{dim} \mathscr{H}^{2}\left(N_{1}\right)+\operatorname{dim} \mathscr{H}^{2}\left(N_{2}\right)+$ $\operatorname{dim} \mathscr{H}^{3}(M)$, provided that $h^{2,1}\left(N_{1}\right)=h^{2,1}\left(N_{2}\right)=h^{3,1}(M)=0$.

Proof. This is a repeated application of Theorem 3.5. Indeed, by virtue of that theorem meromorphic 2-forms on $N_{1}$ with prescribed residue $\theta \in \mathscr{H}^{1}(\Sigma)$ along $\Sigma$ differ by holomorphic 2-forms on $N_{1}$, i.e. by elements of $\mathscr{H}^{2}\left(N_{1}\right)$. Every such 2form is the residue of a meromorphic 3-form on $M$ with poles along $N_{1} \cup N_{2}$. The 3-forms with equal residue along $N_{1}$ differ by meromorphic 3-forms on $M$ with poles along $N_{2}$, i.e. by the elements of $\mathscr{H}^{3}\left(M \backslash N_{2}\right)$. The latter space is isomorphic to $\mathscr{H}^{2}\left(N_{2}\right) \oplus \mathscr{H}^{3}(M)$ by Theorem 3.5.

To describe the corresponding splittings in the "dual" spaces we need the following 
Definition 3.7. Let $N$ be a complex hypersurface in a complex $n$-dimensional manifold $M$. Denote by $\left\{H_{n}(M, \mathbb{Z}) / \mathbb{Z}[N]\right\}$ the quotient of the homology group $H_{n}(M, \mathbb{Z})$ over the space $\mathbb{Z}[N]$ generated by all $n$-cycles in $M$ coming from the hypersurface $N$.

This quotient space is spanned by the homology classes of all $n$-cycles in $M$ which are orthogonal to $\mathbb{Z}[N]$ with respect to the intersection bilinear form in $H_{n}(M, \mathbb{Z})$. Such cycles in $M$ can be "moved away" from $N$. The quotient may have torsion.

Theorem 3.8 (c.f. [T, Cl]). The homology groups of $N_{i} \backslash \Sigma, i=1,2$ and $M \backslash$ $\left(N_{1} \cup N_{2}\right)$ have the following decomposition in terms of the homology groups of $M, N_{1}, N_{2}, \Sigma$ and of the cycles being images under the Leray operator $\delta$ :

$$
\begin{gathered}
H_{2}\left(N_{i} \backslash \Sigma, \mathbb{Z}\right)=\left\{H_{2}\left(N_{i}, \mathbb{Z}\right) / \mathbb{Z}[\Sigma]\right\} \oplus \delta\left(H_{1}(\Sigma, \mathbb{Z})\right), \\
H_{3}\left(M \backslash\left(N_{1} \cup N_{2}\right), \mathbb{Z}\right)=H_{3}(M, \mathbb{Z}) \oplus \sum_{i=1}^{2}\left\{\delta\left(H_{2}\left(N_{i}, \mathbb{Z}\right) / \mathbb{Z}[\Sigma]\right\}\right) \\
\oplus \delta^{2}\left(H_{1}(\Sigma, \mathbb{Z})\right),
\end{gathered}
$$

provided that $H_{3}\left(N_{1}\right)=H_{3}\left(N_{2}\right)=0$. In particular, the maps $\delta$ and $\delta^{2}$ in this case are monomorphisms.

Proof of $(3.3 a)$. The long exact sequence of relative homology groups for a pair $\Sigma \subset N$ gives

$$
H_{2}(\Sigma, \mathbb{Z}) \rightarrow H_{2}(N, \mathbb{Z}) \rightarrow H_{2}(N, \Sigma ; \mathbb{Z}) \rightarrow H_{1}(\Sigma, \mathbb{Z}) \rightarrow H_{1}(N, \mathbb{Z})=0 .
$$

Using Definition 3.7 for the embedding $H_{2}(\Sigma, \mathbb{Z}) \rightarrow H_{2}(N, \mathbb{Z})$ one can rewrite it as

$$
0 \rightarrow\left\{H_{2}(N, \mathbb{Z}) / \mathbb{Z}[\Sigma]\right\} \rightarrow H_{2}(N, \Sigma ; \mathbb{Z}) \rightarrow H_{1}(\Sigma, \mathbb{Z}) \rightarrow 0
$$

Note that the space $H_{2}(N, \Sigma ; \mathbb{Z})$ is dual to $H_{2}(N \backslash \Sigma, \mathbb{Z})$. Hence using representation of the quotient $\left\{H_{2}(M, \mathbb{Z}) / \mathbb{Z}[N]\right\}$ by the 2-cycles in $N$ which do not meet $\Sigma$ and by passing to the dual spaces one obtains the exact sequence

$$
0 \leftarrow\left\{H_{2}(N, \mathbb{Z}) / \mathbb{Z}[\Sigma]\right\} \leftarrow H_{2}(N \backslash \Sigma, \mathbb{Z}) \leftarrow H_{1}(\Sigma, \mathbb{Z}) \leftarrow 0,
$$

where the embedding $H_{1}(\Sigma, \mathbb{Z}) \rightarrow H_{2}(N \backslash \Sigma, \mathbb{Z})$ is nothing else but the Leray map $\delta$. It completes the proof of (3.3a).

Notice, that there is a natural map $\left\{H_{2}\left(N_{i}, \mathbb{Z}\right) / \mathbb{Z}[\Sigma]\right\} \rightarrow H_{2}\left(N_{i} \backslash \Sigma, \mathbb{Z}\right)$ : every 2-cycle in $N$ which does not intersect $\Sigma$ can be regarded as an element of the homology group $H_{2}\left(N_{i} \backslash \Sigma\right)$. The embedding in the opposite direction $H_{2}\left(N_{i} \backslash \Sigma, \mathbb{Z}\right) \rightarrow$ $\mathrm{H}_{2}\left(N_{i}, \mathbb{Z}\right)$ is tautological. The formula $(3.3 \mathrm{~b})$ is proved similarly, see details in [T].

In the decomposition (3.3b) we will call $H_{3}(M, \mathbb{Z}) \oplus \sum_{i=1}^{2}\left\{\delta\left(H_{2}\left(N_{l}, \mathbb{Z}\right) / \mathbb{Z}[\Sigma]\right\}\right)$ the subspace of non-Leray cycles in $H_{3}\left(M \backslash\left(N_{1} \cup N_{2}\right), \mathbb{Z}\right)$. The following theorem provides us with a source of manifolds satisfying a certain quantization condition described in the next section. 
Theorem 3.9. For Kähler manifolds $\Sigma, N_{1}, N_{2}, M$ with $H^{3}\left(N_{1}\right)=H^{3}\left(N_{2}\right)=$ $H^{3,1}(M)=0$ the subspace of 3-forms $\eta \in \mathscr{H}^{3}\left(M \backslash\left(N_{1} \cup N_{2}\right)\right)$ with vanishing integrals over non-Leray 3-cycles in $H_{3}\left(M \backslash\left(N_{1} \cup N_{2}\right), \mathbb{Z}\right)$ has dimension at least $\mathbf{g}=\operatorname{dim}_{\mathbb{C}} \mathscr{H}^{1}(\Sigma)$.

Proof. The number of constraints on the meromorphic forms is majorated by the dimension of the space $H_{3}(M, \mathbb{Z}) \oplus \sum_{i=1}^{2} \delta\left(H_{2}\left(N_{i}, \mathbb{Z}\right)\right)$. Notice, that each summand in this sum imposes as many nontrivial conditions on $\eta$ 's as the dimension of the corresponding space of holomorphic forms $\mathscr{H}^{2}\left(N_{i}\right)$ or $\mathscr{H}^{3}(M)$.

For instance, decompose the space of complex cycles $H_{3}(M, \mathbb{C})$ on the Kähler manifold $M$ into the Hodge subspaces $H_{3}=H_{3,0} \oplus H_{2,1} \oplus H_{1,2} \oplus H_{0,3}$ Poincaré dual to the corresponding spaces of differential forms. Then the integrals over all $(3,0)$-cycles with complex coefficients spanning the space $H_{3,0}(M)$ prescribe exactly $\operatorname{dim} H_{3,0}(M)=\operatorname{dim} \mathscr{H}^{3}(M)$ conditions. On the other hand, the integrals of meromorphic forms over an arbitrary 3-cycle representing a homology class from other summands are automatically zero: by the Poincaré duality $\int_{\sigma} \eta=\int_{M} \eta \wedge \alpha_{\sigma}=0$, where $\alpha_{\sigma} \in H^{1,2} \oplus H^{2,1} \oplus H^{3,0}$ is dual to the cycle $\sigma \in H_{2,1} \oplus H_{1,2} \oplus H_{0,3}$, while the meromorphic form $\eta$ has the type $(3,0)$.

Notice that the integral of a differential form over any cycle of finite order, i.e. generating torsion in $\mathscr{H}^{3}\left(M \backslash\left(N_{1} \cup N_{2}\right)\right)$ is automatically zero.

Thus the dimension of the meromorphic 3-forms on $M$ furnishing the required constraints is not less than the dimension of holomorphic 1-forms on $\Sigma$ by Corollary 3.6.

\section{Four-Dimensional Realization of Central Extensions}

4.1. The two-dimensional current algebra as a quotient. Consider an embedding of the complex curve $\Sigma$ into a complex compact surface $N$ with $h^{2,1}(N)=0$ (i.e., into a simply connected surface $\left.H_{1}(N)=H_{3}(N)=0\right)$. Let $\mathscr{G}^{N}=C^{\infty}(N, \mathscr{G})$ be the Lie algebra of all smooth maps from $N$ to a simple complex Lie algebra $\mathscr{G}$ with the pointwise commutator. Define

$$
\mathscr{G}_{\Sigma}^{N}:=\left\{X \in \mathscr{G}^{N}|X|_{\Sigma}=0\right\}
$$

to be a subalgebra of maps "based" at $\Sigma \subset N$.

Now the two dimensional current Lie algebra $\mathscr{G}^{\Sigma}$ can be viewed as a quotient algebra $\mathscr{G}^{\Sigma} \simeq \mathscr{G}^{N} / \mathscr{G}_{\Sigma}^{N}$. Denote by $G^{N}$ the current group of all smooth contractible maps of $N$ into $G$, and by $G_{\Sigma}^{N}$ the corresponding subgroup

$$
G_{\Sigma}^{N}:=\left\{g \in G^{N}|g|_{\Sigma}=i d\right\} .
$$

They are related by the same identity $G^{\Sigma} \simeq G^{N} / G_{\Sigma}^{N}$, since all maps of $\Sigma$ into $G$ (i.e. all elements of $G^{\Sigma}$ ) are contractible for a simple simply connected group $G: \pi_{2}(G)=H^{2}(G)=0$.

The next step is to define the central extensions of the Lie algebra $\mathscr{G}^{N}$ and the Lie group $G^{N}$.

For this purpose, given any holomorphic 1-form $\theta \in \mathscr{H}^{1}(\Sigma)$ on $\Sigma$ we pick out a meromorphic 2-form $\omega \in \mathscr{H}^{2}(N \backslash \Sigma)$ on $N$ which has a polar set of the first order on $\Sigma$ and such that $\theta=\operatorname{res} \omega$. 
As we mentioned above, for any meromorphic 2-form on $N$ with poles on $\Sigma$ the residue is a holomorphic 1-form on $\Sigma$. Conversely, if $H_{3}(N)=0$ then every holomorphic 1-form on $\Sigma$ is the residue of some meromorphic 2-form on $N$ by virtue of the exact sequence (3.2). The kernel of the residue map consists of all holomorphic 2-forms on $N$ (and it is trivial, for instance, for $N=\mathbb{C} P^{2}$ ).

Definition 4.1. Let us fix a lifting

$$
l_{1}: \mathscr{H}^{1}(\Sigma) \rightarrow \mathscr{H}^{2}(N \backslash \Sigma, \mathbb{C})
$$

of the holomorphic 1-forms on $\Sigma$ to the meromorphic 2-forms on $N$ with poles on $\Sigma$, such that Res $\circ \imath_{1}=I d$. We define the central extension $\hat{\mathscr{G}}^{N}$ with the center $\mathscr{H}^{1}(\Sigma)^{*}=\left(\mathscr{H}^{2}(N \backslash \Sigma) / \mathscr{H}^{2}(N)\right)^{*}$ by means of the cocycle

$$
\langle C(X, Y), \theta\rangle=C_{l_{1}(\theta)}(X, Y),
$$

where the complex-valued two-cocycle $C_{\omega}(X, Y)$ associated to a meromorphic 2form $\omega \in \mathscr{H}^{2}(N \backslash \Sigma, \mathbb{C})$ is

$$
C_{\omega}(X, Y)=\frac{1}{2 \pi i} \int_{N} \operatorname{tr}(d X \wedge d Y) \wedge \omega .
$$

The proof of the cocycle identity for $C$ literally repeats the 1-dimensional case. It is clear that the central extension is independent of the choice of the embedding $l_{1}$. Evidently, the 2-cocycle depends only on the cohomology class of $\omega$ in $\mathscr{H}^{2}(N \backslash \Sigma, \mathbb{C})$.

Proposition 4.2. Let $\Sigma$ be embedded into a complex surface $N$ with $h^{2,1}(N)=0$. Then the Lie algebra $\mathscr{G}_{\Sigma}^{N}$ is an ideal in $\hat{\mathscr{G}}^{N}$. The quotient algebra $\hat{\mathscr{G}}^{N} / \mathscr{G}_{\Sigma}^{N}$ is naturally isomorphic to the central extension $\hat{\mathscr{G}}^{\Sigma}$ of the current algebra on $\Sigma$, defined by the cocycle (2.1).

Proof. It is an immediate corollary of Theorem 3.4 applied to the manifold $N$ and the 4-form $\mu=\operatorname{tr}(d X \wedge d Y) \wedge \omega$ whose primitive 3-form $\alpha=d^{-1} \omega$ is taken to be $\alpha=\operatorname{tr}(X d Y) \wedge \omega$ :

$$
C_{\omega}(X, Y)=\frac{1}{2 \pi i} \int_{N} \operatorname{tr}(d X \wedge d Y) \wedge \omega=-\int_{\Sigma} \operatorname{res}(\operatorname{tr}(X d Y) \wedge \omega)=\int_{\Sigma} \operatorname{tr}(X d Y) \wedge \theta .
$$

This shows that $\mathscr{G}_{\Sigma}^{N}$ is an ideal in $\hat{\mathscr{G}}^{N}$. The quotient algebra is isomorphic to $\hat{\mathscr{G}}^{\Sigma}$, see Definition 2.1.

Note that though the realization described goes through for any $C^{\infty}$ form $\omega$ on $N \backslash \Sigma$ and without the assumption $h^{2,1}(N)=0$, the condition on $\omega$ to be meromorphic makes the construction canonical.

Remark 4.3. A stimulus for our consideration was a similar cocycle expression $\int \operatorname{tr}(d X \wedge d Y) \wedge \omega$ with a Kähler form $\omega$ appeared in the work [LMNS, NS]. However, the orbit structure of the algebra with a Kähler form is rather obscure. Already for an abelian group $G$ the coadjoint orbits of the corresponding current group are at least as complicated as for the group of volume-preserving diffeomorphism of 
a three-dimensional manifold. In our construction, the de Rham theory of smooth forms is replaced by the Leray residue theory, which is more appropriate for the complex-valued setting. The corresponding group $\hat{G}^{\Sigma}$ comprises a nice orbit structure $([\mathrm{EF}, \mathrm{EK}])$, that indicates the existence of interesting representation theory.

4.2. The extension of the current groups. Similarly to the classical WZNW-construction of affine groups, we proceed by defining the group extension $\hat{G}^{N}$ corresponding to the Lie algebra $\hat{\mathscr{G}}^{N}$ of currents on a complex surface $N$ containing the curve $\Sigma$.

Definition 4.4. The central extension $\hat{G}^{N}$ of the Lie group $G^{N}$ with the center $\mathscr{H}^{1}(\Sigma)^{*} / L$, where $L$ is a certain lattice, is given by the following 2-cocycle

$$
\left\langle\Gamma\left(g_{1}, g_{2}\right), \theta\right\rangle=\Gamma_{l_{1}(\theta)}\left(g_{1}, g_{2}\right)
$$

defined for any two group currents $g_{1}, g_{2} \in G^{N}=C^{\infty}(N, G)$ modulo the lattice L. Here for any meromorphic 2-form $\omega$ on $N$ with polar set $\Sigma$ of the first order

$$
\Gamma_{\omega}\left(g_{1}, g_{2}\right):=\frac{1}{4 \pi i} \int_{N} \operatorname{tr}\left(g_{1}^{-1} d g_{1} \wedge d g_{2} g_{2}^{-1}\right) \wedge \omega .
$$

One can check directly the cocycle property as in the WZNW classical case. Verification of the fact that the Lie algebra corresponding to the Lie group $\hat{G}^{N}$ coincides with $\hat{\mathscr{G}}^{N}$ is immediate. Note that the definition makes sense for a trivial lattice $L=0$, defining a central extension of $G^{N}$ by the whole space $\mathscr{H}^{1}(\Sigma)^{*}$. However, we will see that in order to define a quotient of $\hat{G}^{N}$ over $G_{\Sigma}^{N}$ the lattice $L$ necessarily contains the lattice of periods $H_{1}(\Sigma, \mathbb{Z})$.

Recall that the subgroup $G_{\Sigma}^{N}$ of "based" maps is defined as

$$
G_{\Sigma}^{N}=\left\{g \in C^{\infty}(N, G)|g|_{\Sigma}=i d\right\} .
$$

Theorem 4.5. Let $\Sigma$ and $N$ be as in Proposition 4.2. Then there is an embedding of the group $G_{\Sigma}^{N}$ into the group $\hat{G}^{N}$ as a normal subgroup such that the corresponding quotient is isomorphic to the group $\hat{G}^{\Sigma}$ of currents on the complex curve $\Sigma$ centrally extended by means of the Jacobian of the curve.

The proof of this theorem occupies the rest of this section. The conditions $h^{2,1}(N)=h^{2,1}(\tilde{\Sigma})=0$ are satisfied for simply connected manifolds $N$ and $\tilde{\Sigma}$. This is a counterpart of simply connectedness of the disks $D$ and $D^{\prime}$ in the classical case, see Sect. 1 . Note that by passing from the holomorphic category to the $C^{\infty}$-category one can drop this requirement in Proposition 4.2 and Theorem 4.5.

We will be looking for the homomorphism $G_{\Sigma}^{N} \rightarrow \hat{G}^{N}$ of the type $g \mapsto(g, \Lambda(g))$, where $g \in G_{\Sigma}^{N}$, and $\Lambda(g) \in \mathscr{H}^{1}(\Sigma)^{*}$ "resolves" the 2-cocycle $\Gamma$, namely

$$
\Lambda\left(g_{1} g_{2}\right)-\Lambda\left(g_{1}\right)-\Lambda\left(g_{2}\right)=\Gamma\left(g_{1}, g_{2}\right) .
$$

To achieve this we need to move one dimension up as in the classical case, though now we are counting the complex dimensions. 
4.3. Resolution of the cocycle. First we embed the complex surface $N$ into a 3dimensional complex manifold $M$ and define the curve $\Sigma$ as a transversal intersection of $N$ and of some nonsingular complex surface $\tilde{\Sigma}$ in $M$. We demand that the manifolds $\tilde{\Sigma}, N$ and $M$ obey the assumptions of Theorem 3.5: the Hodge dimensions $h^{2,1}(\tilde{\Sigma})=h^{2,1}(N)=h^{3,1}(M)=0$ are trivial. These conditions are technical and related to the consideration of holomorphic and meromorphic forms. They are not required in the $C^{\infty}$ category.

Pursuing the same strategy as before, fix an embedding

$$
l_{2}: \mathscr{H}^{2}(N \backslash \Sigma, \mathbb{C}) \rightarrow \mathscr{H}^{3}(M \backslash(N \cup \tilde{\Sigma}), \mathbb{C})
$$

such that $\operatorname{Res} \circ l_{2}=I d$. In other words, given meromorphic 2-form $\omega$ on $N$ we pick up a meromorphic 3-form $\eta$ in $M \backslash(N \cup \tilde{\Sigma})$ with a pole of the first order on (the nonsingular part of) $\quad N \cup \tilde{\Sigma}$, such that on the manifold $N$ the 2-form res $\eta$ coincides with the 2 -form $\omega$. We do not require anything for the restriction $\operatorname{res} \eta_{\tilde{\Sigma}}$.

Two different meromorphic "extensions" of the 2-form $\omega$ on $N$ differ by a meromorphic 3-form on $M$ with the polar set $\tilde{\Sigma}$. Say, for $M=\mathbb{C} P^{3}$ one has as many extensions as holomorphic 2 -forms on $\tilde{\Sigma}$, according to the exact sequence (3.2).

Given current $g$ we define the quantity $\Lambda(g)$ assuming values in $\mathscr{H}^{1}(\Sigma)^{*}$ by the relation $\langle\Lambda(g), \theta\rangle=\Lambda_{l^{2}(\theta)}(g)$, where the embedding $l^{2}:=l_{2} \circ l_{1}$ is the composition of $l_{2}$ and $l_{1}$.

Definition 4.6. Consider any extension $\bar{g}$ of a current $g \in C^{\infty}(N, G)$ from the complex surface $N$ to the ambient space $M$, such that $\left.\bar{g}\right|_{\Sigma}=i d$. We define the quantity $\Lambda(g)$ with values in $\mathscr{H}^{1}(\Sigma)^{*}$ by the relation

$$
\langle\Lambda(g), \theta\rangle=\Lambda_{l^{2}(\theta)}(g),
$$

where

$$
\Lambda_{\eta}(g)=-\frac{1}{24 \pi^{2}} \int_{M} \operatorname{tr}\left(\bar{g}^{-1} d \bar{g}\right)^{3} \wedge \eta
$$

and $\eta \in \mathscr{H}^{3}(M \backslash(N \cup \tilde{\Sigma}))$.

Such an extension $\bar{g}$ exists due to our assumption on triviality of the homotopy type of the maps $g: N \rightarrow G$.

As in the classical case, this definition may depend on the choice of an extension $\bar{g}$, and strictly speaking is not well-defined for a given current $g$. We will show that the ambiguity of the definition of $\Lambda(g)$ in the choice of an extension $\bar{g}$ results in shifts by elements from a certain lattice $L$.

Let us assume for a moment that this is so. Then we have the following

Proposition 4.7 (cf. 4.5). Let $\Sigma$ and $N$ be as in Proposition 4.2. Assume also that $\Lambda(g)$ is well-defined modulo a lattice $L$ in $\mathscr{H}^{1}(\Sigma)^{*}$. Let $\hat{G}^{N}$ be the central extension of $G^{N}$ by means of the quotient $\mathscr{H}^{1}(\Sigma)^{*} / L$ defined by the 2-cocycle $\Gamma\left(g_{1}, g_{2}\right)$. Then the group $G_{\Sigma}^{N}$ is embedded into $\hat{G}^{N}$ as a normal subgroup, and the Lie algebra of the quotient group $\hat{G}^{\Sigma}=\hat{G}^{N} / G_{\Sigma}^{N}$ is isomorphic to $\hat{\mathscr{G}}^{\Sigma}$. 
Proof. Let us verify here that the embedding $G_{\Sigma}^{N} \ni g \mapsto(g, \Lambda(g)) \in \hat{G}^{N}$ is a group homomorphism. Indeed,

$$
\begin{aligned}
\Lambda_{\eta}\left(g_{1} g_{2}\right)= & -\frac{1}{24 \pi^{2}} \int_{M} \operatorname{tr}\left(\left(\bar{g}_{1} \bar{g}_{2}\right)^{-1} d\left(\bar{g}_{1} \bar{g}_{2}\right)\right)^{3} \wedge \eta \\
= & -\frac{1}{24 \pi^{2}} \int_{M} \operatorname{tr}\left(\bar{g}_{2}^{-1} \bar{g}_{1}^{-1}\left(d \bar{g}_{1}\right) \bar{g}_{2}+\bar{g}_{2}^{-1} d \bar{g}_{2}\right)^{3} \wedge \eta \\
= & \Lambda_{\eta}\left(g_{1}\right)+\Lambda_{\eta}\left(g_{2}\right) \\
& -\frac{1}{8 \pi^{2}} \int_{M} \operatorname{tr}\left(\left(\bar{g}_{1}^{-1} d \bar{g}_{1}\right)^{2} d \bar{g}_{2} \bar{g}_{2}^{-1}+\bar{g}_{1}^{-1} d \bar{g}_{1}\left(d \bar{g}_{2} \bar{g}_{2}^{-1}\right)^{2}\right) \wedge \eta \\
= & \Lambda_{\eta}\left(\bar{g}_{1}\right)+\Lambda_{\eta}\left(\bar{g}_{2}\right)+\frac{1}{8 \pi^{2}} \int_{M} d\left(\operatorname{tr}\left(\bar{g}_{1}^{-1} d \bar{g}_{1} \wedge d \bar{g}_{2} \bar{g}_{2}^{-1}\right)\right) \wedge \eta .
\end{aligned}
$$

Applying Leray Theorem 3.4 we transform the last term to the form

$$
\begin{aligned}
\frac{1}{4 \pi i} \int_{N \cup \tilde{\Sigma}} \operatorname{tr}\left(\bar{g}_{1}^{-1} d \bar{g}_{1} \wedge d \bar{g}_{2} \bar{g}_{2}^{-1}\right) \wedge \operatorname{res} \eta & =\frac{1}{4 \pi i} \int_{N} \operatorname{tr}\left(g_{1}^{-1} d g_{1} \wedge d g_{2} g_{2}^{-1}\right) \wedge \omega \\
& =\Gamma_{\omega}\left(g_{1}, g_{2}\right) .
\end{aligned}
$$

Here the integral over $N \cup \tilde{\Sigma}$ descends to the integral over $N$ due to the following. The currents $\bar{g}_{1}$ and $\bar{g}_{2}$ are equal to $i d \in G$ on the surface $\tilde{\Sigma}$. Hence the two form $\beta:=\operatorname{tr}\left(\bar{g}_{1}^{-1} d \bar{g}_{1} \wedge d \bar{g}_{2} \bar{g}_{2}^{-1}\right)$ vanishes on $\tilde{\Sigma}$, and so does the 3 -form $d \beta$. Therefore, the singularity of the 6 -form $d \beta \wedge \eta$ is removable on $\tilde{\Sigma}$, and the residue theorem reduces the integral over $M$ to that over $N$. Proposition 4.7 is proved.

4.4. The lattice of cocycle values. To complete the proof of Theorem 4.5 we need to analyse the ambiguity in the definition of $\Lambda(g)$.

Let $\bar{g}$ and $\overline{\bar{g}}$ be two different extensions of a current $g$, such that $\bar{g},\left.\overline{\bar{g}}\right|_{\tilde{\Sigma}}=i d$. Then $\overline{\bar{g}}=h \bar{g}$ for some current $h \in C^{\infty}(M, G)$ such that $\left.h\right|_{\tilde{\Sigma} \cup N}=i d$. We denote the set of all currents obeying these conditions by $G_{\tilde{\Sigma} \cup N}^{M}$. The same argument as in the proof of Proposition 4.7 shows that $\Lambda(\overline{\bar{g}})=\Lambda(h \bar{g})=\Lambda(h)+\Lambda(\bar{g})+\Gamma(h, \bar{g})$. However, now $\Gamma(h, \bar{g})=0$, since the restriction of the 1 -form $h^{-1} d h$ to $\tilde{\Sigma} \cup N$ vanishes due to the requirement on the current $h$ that $\left.h\right|_{\tilde{\Sigma} \cup N}=i d$.

Hence, it remains to determine the set $L$ of possible values of $\Lambda(h)$ for all $h \in G_{\tilde{\Sigma} \cup N^{*}}^{M}$. It is clear from the above ambiguity of $\Lambda(h)$ that $L$ is an abelian subgroup of $\mathscr{H}^{1}(\Sigma)^{*}$. Given current $h$ we rewrite $\Lambda_{\eta}(h)$ for an arbitrary 3 -form $\eta$ as the integral over the 3-cycle $\Delta_{h}$, Poincare dual in $M$ to the smooth differential 3-form $-\frac{1}{24 \pi^{2}} \operatorname{tr}\left(h^{-1} d h\right)^{3}$ :

$$
\Lambda_{\eta}(h)=-\frac{1}{24 \pi^{2}} \int_{M} \operatorname{tr}\left(h^{-1} d h\right)^{3} \wedge \eta=\int_{\Delta_{h}} \eta .
$$


Proposition 4.8. The 3-cycle $\Delta_{h}$ Poincaré dual to the 3-form $-\frac{1}{24 \pi^{2}} t r\left(h^{-1} d h\right)^{3}$ defines an element of the homology group $H_{3}(M \backslash(\tilde{\Sigma} \cup N), \mathbb{Z})$ with integer coefficients. Moreover, every Leray 3-cycle in $M \backslash(\tilde{\Sigma} \cup N)$ can be represented by a cycle $\Delta_{h}$ for some $h \in G_{\tilde{\Sigma} \cup N}^{M}$.

Proof. Indeed, recall that $h$ is a map $M \rightarrow G$, and the group $G$ has one-dimensional group $H^{3}(G, \mathbb{Z})$. The 3 -form $-\frac{1}{24 \pi^{2}} \operatorname{tr}\left(h^{-1} d h\right)^{3}$ is the pull-back to $M$ of the generator of $H^{3}(G, \mathbb{Z})$ like in the classical case, see Proposition 1.4. Furthermore, the form $-\frac{1}{24 \pi^{2}} \operatorname{tr}\left(h^{-1} d h\right)^{3}$ vanishes on $\tilde{\Sigma} \cup N \subset M$. Hence it is Poincaré dual to some 3-cycle $\Delta_{h}$ whose support is disjoint from $\tilde{\Sigma} \cup N$. Thus the cycle $\Delta_{h}$ can be considered as an element in $H_{3}(M \backslash(\tilde{\Sigma} \cup N), \mathbb{Z})$.

Moreover, consider an arbitrary embedded curve $\sigma$ on the Riemann surface $\Sigma$ which represents an element of $H_{1}(\Sigma, \mathbb{Z})$. Then its Leray image $\delta^{2} \sigma$ is an embedded three-torus $T^{3} \in M \backslash(\tilde{\Sigma} \cup N)$. Let $U\left(T^{3}\right) \simeq T^{3} \times B^{3}$ be a tubular neighborhood of the torus. Impose the condition on $h$ to send the boundary of the three-dimensional ball $B^{3}$ (and the whole complement $M \backslash U\left(T^{3}\right)$ of the neighborhood) to $i d \in G$. One may think of $h$ as a map $T^{3} \times S^{3} \rightarrow G$. Then the desired $h$ is invariant along $T^{3}$ and it sends $S^{3}$ with degree 1 to a spheroid in $G$ generating $\pi_{3}(G)$. Indeed, the support of the corresponding three-form $-\frac{1}{24 \pi^{2}} \operatorname{tr}\left(h^{-1} d h\right)^{3}$ is contained in $U\left(T^{3}\right)$. Moreover, one can see that the integral of this form over any 3-cycle in $M \backslash(\tilde{\Sigma} \cup N)$ is equal to the intersection number of such a cycle with $T^{3}$. By definition, this means that $T^{3}=\delta^{2} \sigma$ is Poincare dual to the 3 -form.

Proposition 4.8 shows that the range of $\Lambda(h)$ in $\mathscr{H}^{1}(\Sigma)^{*}$ contains the lattice $H_{1}(\Sigma, \mathbb{Z})$. Indeed, the Leray operator $\delta^{2}$ embeds the set $H_{1}(\Sigma, \mathbb{Z})$ as a sublattice of $H_{3}(M \backslash(\tilde{\Sigma} \cup N), \mathbb{Z})$, since it is injective (see Sect. 3.4), while the integrals of $\eta$ taken over the Leray cycles $\delta^{2} \sigma$ descend to the integrals of res $\eta$ over $\sigma$.

Impose the following quantization condition on the embedding $l^{2}: \mathscr{H}^{1}(\Sigma, \mathbb{C}) \rightarrow$ $\mathscr{H}^{3}(M \backslash(\tilde{\Sigma} \cup N), \mathbb{C})$. We fix an arbitrary sublattice in $H_{3}(M \backslash(\tilde{\Sigma} \cup N), \mathbb{Z})$ complementary to $\delta^{2}\left(H_{1}(\Sigma, \mathbb{Z})\right)$, and we require the image of $l_{2} \circ l_{1}$ to belong to the subspace of the meromorphic 3-forms $\eta \in \mathscr{H}^{3}(M \backslash(\tilde{\Sigma} \cup N), \mathbb{C})$ which vanish on the chosen sublattice. The complex dimension of the vector space spanned by such $\eta$ 's is at least $\operatorname{dim}_{\mathbb{C}} \mathscr{H}^{1}(\Sigma)=\mathbf{g}$ as Theorem 3.7 shows.

It immediately implies that value of $\Lambda(h)$ on arbitrary three-cycles from $H_{3}(M \backslash(\tilde{\Sigma} \cup N), \mathbb{Z})$ will be necessarily of the form

$$
\int_{\delta^{2} \sigma} \eta=(2 \pi i)^{2} \int_{\sigma} \operatorname{res}^{2} \eta=(2 \pi i)^{2} \int_{\sigma} \theta,
$$

where $\sigma \in H_{1}(\Sigma, \mathbb{Z})$. Thus the values of $\Lambda(h)$ form the lattice $L=H_{1}(\Sigma, \mathbb{Z})$.

In other words, for the described embeddings $l^{2}: \mathscr{H}^{1}(\Sigma, \mathbb{C}) \rightarrow \mathscr{H}^{3}(M \backslash(\tilde{\Sigma} \cup$ $N), \mathbb{C})$ the projection of the dual spaces send the lattice $H_{3}(M \backslash(\tilde{\Sigma} \cup N), \mathbb{Z})$ to the lattice $H_{1}(\Sigma, \mathbb{Z})$, that gives the desired realization of $\hat{G}^{\Sigma}$. This completes the proof of Theorem 4.5.

4.5. Examples and generalizations. The simplest nontrivial example of our theorem corresponds to an elliptic curve $\Sigma$. In this case we can embed it into $N=\mathbb{C} P^{2}$ for which $H_{1}(N)=0$. Moreover, we can realize $\Sigma$ as a transverse intersection of a cubic surface $\tilde{\Sigma}$ and $\mathbb{C} P^{2}$ inside $\mathbb{C} P^{3}$ (note that $h^{2,1}\left(\mathbb{C} P^{2}\right)=h^{2,1}(\tilde{\Sigma})=h^{3,1}\left(\mathbb{C} P^{3}\right)=0$ ). 
Corollary 4.9. Let $\Sigma$ be an elliptic curve realized as a transverse intersection $\tilde{\Sigma} \cap$ $\mathbb{C} P^{2}$ inside $\mathbb{C} P^{3}$. Then $\hat{G}^{\Sigma} \simeq \hat{G}^{\mathbb{C} P^{2}} / \hat{G}_{\Sigma}^{\mathbb{C} P^{2}}$.

For a complex curve of an arbitrary genus it is known that it admits an embedding in $\mathbb{C} P^{3}$, but, generally speaking, only immersions in $\mathbb{C} P^{2}$, see [ACGH]. To obtain a complex surface $N$ with $H_{1}(N)=H_{3}(N)=0$ one can resolve double points in $\mathbb{C} P^{2}$. Notice, that not all complex curves are complete intersections of algebraic surfaces in $\mathbb{C} P^{3}$ (see e.g. $[\mathrm{ACGH}]$ ).

Remark 4.10. One can avoid the use of meromorphic Leray theory outlined in Sect. 3.4 by imposing the same quantization condition on more general liftings

$$
\begin{aligned}
& \iota_{1}: \mathscr{H}^{1}(\Sigma) \rightarrow Z^{2}(N \backslash \Sigma, \mathbb{C}), \\
& \iota_{2}: Z^{2}(N \backslash \Sigma, \mathbb{C}) \rightarrow Z^{3}(M \backslash(N \cup \tilde{\Sigma}), \mathbb{C}),
\end{aligned}
$$

where $Z^{2}$ and $Z^{3}$ denote the spaces of smooth closed two- and three- forms with poles of the first order. It is remarkable that these embeddings can be realized within the spaces of meromorphic forms, provided that certain conditions on the manifolds are obeyed.

Remark 4.11. It follows from our construction that the central extension of $G^{\Sigma}$ by means of the Jacobian variety $\mathscr{J}$ is "maximal possible" in the category of Lie groups with the Lie algebra $\hat{\mathscr{G}}^{\Sigma}$. In fact, one can show that it is universal in this class of Lie groups. Other extensions correspond to a choice of lattices $L \subset \mathscr{H}^{1}(\Sigma)^{*}$ which contain $H_{1}(\Sigma, \mathbb{Z})$ and are obtained via the natural projection $\mathscr{J} \rightarrow \mathscr{H}^{\mathrm{l}}(\Sigma)^{*} / L$.

In particular, we can weaken the conditions on the manifolds $M$ and $N$ in Theorem 4.6 or on the embedding $l_{1} \circ l_{2}$ only to ensure that the set of values of $\Lambda(h)$ is a lattice $L \subset \mathscr{H}^{1}(\Sigma)^{*}$. Then our construction still yields a central extension of the group $G^{\Sigma}$ with the Lie algebra $\hat{\mathscr{G}}^{\Sigma}$.

The quotient group $L / H_{1}(\Sigma, \mathbb{Z})$ (or the corresponding index $\left[L: H_{1}(\Sigma, \mathbb{Z})\right]$ ) can be viewed as an analogue of the level in the classical case. Recalling the fundamental importance of level 1 representations of affine Lie groups of ADE types, one wonders whether level 1 representations of central extensions of two-dimensional current groups will play a similar role in representation theory of these groups.

\section{Generalizations to Current Algebras on Punctured Complex Curves and Further Perspectives}

5.1. Current algebras on punctured curves. One can almost literally generalize the above constructions to the case of punctured Riemann surfaces. Holomorphic 1-forms on $\Sigma$ are replaced by meromorphic ones with prescribed poles. Their extensions to the spaces $N$ and $M$ also demand the consideration of meromorphic forms with given orders of poles at arrangements of hyperplanes. In this section we just mention several necessary changes in the described realization to include punctures on $\Sigma$ into the general scheme.

Let $\theta$ be a meromorphic form on a complex curve $\Sigma$ with poles at the set $\Pi=\left\{p_{1}, \ldots, p_{r}\right\}$. Recall that the algebra $\mathscr{G}_{\Pi}^{\Sigma}$ consists of $\mathscr{G}$-valued functions on $\Sigma$ 
assuming zero values at each point of $\Pi$. The central extension of this algebra is given by the cocycle (2.1).

In order to obtain a four dimensional realization of this algebra we consider an embedding of $\Sigma$ into a complex surface $N$. Moreover, on the surface $N$ we consider a set of complex curves $\Xi:=\Sigma \cup P_{1} \cup \cdots \cup P_{r}$ in a general position such that the intersection of the curve $\Sigma$ with all the rest $P:=\bigcup_{i=1}^{r} P_{i}$ is exactly the set of removed points $\Sigma \cap P=\left\{p_{1}, \ldots, p_{r}\right\}$. (Say, a Riemann sphere with three punctures, crucial for many constructions in CFT, can be seen here as a quadruple of complex lines in $N=\mathbb{C} P^{2}$.) Then for the corresponding current algebras one obtains a natural isomorphism $\mathscr{G}_{\Pi}^{\Sigma} \simeq \mathscr{G}_{P}^{N} / \mathscr{G}_{\Xi}^{N}$ with the evident notations $\mathscr{G}_{N}^{M}:=\{X \in$ $\left.C^{\infty}(M, \mathscr{G})|X|_{N}=0\right\}$.

Furthermore, choose an arbitrary closed 2-form $\omega$ which is $C^{\infty}$-smooth in $N \backslash \Xi$, it has a first order pole along $\Xi$, and res $\left.\omega\right|_{\Sigma}=\theta$. Notice, that poles of $\omega$ at the points $p_{i}$ are of the second order. The 2-cocycle on $\mathscr{G}_{P}^{N}$ is defined by the formulas $(4.1-2)$.

Proposition 5.1 (cf. 4.2). The current algebra $\hat{\mathscr{G}}_{\Pi}^{\Sigma}$ is isomorphic to the following quotient:

$$
\hat{\mathscr{G}}_{\Pi}^{\Sigma} \simeq \hat{\mathscr{G}}_{P}^{N} / \mathscr{G}_{\Xi}^{N}
$$

The proof of the proposition is immediate. Notice, that if instead of $\mathscr{G}_{\Pi}^{\Sigma}$ we deal with a more general algebra of currents on $\Sigma$ with values in nilpotent subalgebras at punctured points $p_{j} \in \Pi$, then the algebras $\mathscr{G}_{P}^{N}$ and $\mathscr{G}_{\Xi}^{N}$ are replaced by the algebras of currents on $N$ assuming values in the same nilpotent subalgebras on the entire curves $P_{j} \subset P$. Intersections of these curves are related to the intersections of the corresponding nilpotent subalgebras.

In the same way one defines the groups $G_{\Xi}^{N}, G_{P}^{N}$ and the central extension $\hat{G}_{P}^{N}$ (see Definition 4.4).

Theorem 5.2 (cf. 4.5). There is an embedding of the group $G_{\Xi}^{N}$ into the group $\hat{G}_{P}^{N}$ as a normal subgroup such that the corresponding quotient is isomorphic to the central extension $\hat{G}_{\Pi}^{\Sigma}$ of the group $G_{\Pi}^{\Sigma}$ of currents on the complex curve $\Sigma$ which vanish at the punctures $\Pi$ on the curve.

To define the embedding $G_{\Xi}^{N} \rightarrow \hat{G}_{P}^{N}$ one has to pass to three complex dimensions. Fix some embedding of the surface $N$ into a complex three-dimensional manifold $M$ and consider a collection of complex two-dimensional submanifolds $\tilde{\Xi}:=\tilde{\Sigma} \cup \tilde{P}_{1} \cup$ $\cdots \cup \tilde{P}_{r}$ in a general position in $M$ such that they cut $N$ along the corresponding curves $\Xi=\Sigma \cup P_{1} \cup \cdots \cup P_{r}$.

Again, specify some closed 3-form $\eta$ which is $C^{\infty}$-smooth in $M \backslash \tilde{\Xi}$, it has a first order pole along $\tilde{\Xi} \cup N$, and res $\left.\eta\right|_{N}=\omega$. Construction of the resolution $\Lambda(g)$ of the 2-cocycle transfers literally to this case.

5.2. Example of punctured sphere: Hyperplane arrangements. Look at the punctured Riemann sphere: $\Sigma=\mathbb{C} P^{1}, N=\mathbb{C} P^{2}$ and $M=\mathbb{C} P^{3}$. In the case of two removed points $(r=2)$ one can consider three complex lines $\Xi=\Sigma \cup P_{1} \cup P_{2}$ in a general position in $N=\mathbb{C} P^{2}$, and extend them to three planes $\tilde{\Xi}=\bigcup_{t=1}^{3} \mathbb{C} P_{i}^{2}$ in 
$\mathbb{C P}^{3}$. Then the complement in $\mathbb{C} P^{3}$ to $\tilde{\Xi} \cup N$ has the following third homology group:

$$
H_{3}\left(\mathbb{C} P^{3} \backslash\left(\bigcup_{i=1}^{4} \mathbb{C} P_{i}^{2}\right), \mathbb{Z}\right)=H_{3}\left(\mathbb{C}^{3} \backslash\left(\bigcup_{i=1}^{3} \mathbb{C}_{i}^{2}\right), \mathbb{Z}\right)=\mathbb{Z}
$$

The Leray map establishes the isomorphism of it with the corresponding homology group

$$
H_{1}\left(\Sigma \backslash\left(p_{1} \cup p_{2}\right)\right)=H_{1}\left(\mathbb{C} P^{1} \backslash\left(p_{1} \cup p_{2}\right)\right)=\mathbb{Z},
$$

cf. Theorem 3.8. For the sphere with three punctures (the key picture in CFT constructions) the homology group $H_{1}$ is $\mathbb{Z} \oplus \mathbb{Z}$, while the corresponding group $H_{3}$ is larger.

The case of punctured Riemann surfaces also covers the current groups constructed on smooth complex curves embedded to a complex surface $N$ with self-intersections. Moreover, our construction is self-consistent with respect to degenerations on the boundary of the moduli space of complex curves: the two parts $\Sigma_{1} \cup_{S} \Sigma_{2}$ of a nonsingular complex curve $\Sigma$ squeezed along some cycle $S$ provide both the punctured surface $\Sigma_{1}$ and the auxiliary set $P=\Sigma_{2}$.

5.3. Remarks on representation theory and quantum field theory. The generalization of two dimensional current groups to the case of punctured Riemann surface strongly allude to the parallel with conformal field theory. But in our setting we expect to attach to a punctured Riemann surface a category of representations of the corresponding current groups rather than a vector space of conformal blocks. These categories are in turn supposed to form a two category similar to the modular tensor category in the classical case and it is natural to expect that the latter category is just a Grothendieck ring of the former two category. This allows us to conjecture that construction of representation theory of two dimensional current algebras and groups will provide a categorical analogue of conformal field theory (cf. $[\mathrm{CF}]$ ).

An equally interesting direction is a development of a four dimensional quantum field theory associated to our four dimensional realization of current groups. One should note from our construction that unless we consider the example of a sphere with punctures it is impossible to write a Lagrangian in pure exponential form, because the topological term is defined only up to a lattice in a complex space. Thus the relation of our construction with the proposed in [LMNS] four dimensional models is not yet clear.

Another interesting problem is a generalization of the Chern-Simons-Witten theory [W2] to the complex setting. Following the analogy inspired by our construction Witten's Lagrangean over a compact (real) three-dimensional manifold should be replaced by the following Lagrangean defined on a Calabi-Yau (complex) three-dimensional manifold $M$ :

$$
S=\frac{1}{2} \int_{M} \operatorname{tr}\left(A \wedge \bar{\partial} A+\frac{2}{3} A \wedge A \wedge A\right) \wedge \eta,
$$

where $A$ is a $\mathscr{G}$-valued one-form of type $(0,1)$ and $\eta$ is the holomorphic three-form on $M$. This Lagrangean appears as a low energy limit of open string theory in [W3], where it is argued that this analogue of CSW theory is still finite. Thus it is natural to expect that the complex generalization of Witten's three-dimensional 
topological invariant yields an invariant of Calabi-Yau manifolds. The meromorphic forms appeared in the present paper should play the role of links in real threedimensional manifolds. The above Lagrangean was also discussed in [BCOV] in relation to the Kodaira-Spencer theory, and it is undoubtly encodes deep mathematical structures.

There are evident connections of our realization of the central extension $\hat{G}^{\Sigma}$ with algebraic geometry and complex analysis. Moreover, the analogy of our construction with the WZNW model indicates relations with other areas of mathematics that are not so transparent at the present moment. For example, when $\Sigma$ is an elliptic curve a representation category of the group $\hat{G}^{\Sigma}$ should involve essentially the arithmetic of the elliptic curve in a similar way as the representation category of $\hat{G}^{S^{1}}$ is associated to the arithmetic of the roots of unity.

Acknowledgements. We have benefited a lot from illuminating discussions with A. Todorov. We are deeply obliged to him, to P. Etingof, M. Khovanov and G. Zuckerman for helpful remarks. We thank G. Moore and N. Nekrasov for discussions and the detailed explanation of their work in progress [LMNS] on the real-valued 4-dimensional WZNW-model, and for providing us with the reference [NS]. The research of I.F. and B.K. was supported by the NSF grants DMS-9400908 and DMS-9307086.

\section{References}

[ACGH] Arbarello, E., Cornalba, M. Griffiths, P.A., Harris, J.: Geometry of algebraic curves. Berlin, Heidelberg, 1985

[BCOV] Bershadsky, M., Cecotti, S., Ooguri, H., Vafa, C.: Kodaira-Spencer theory of gravity and exact results for quantum string amplitudes. Comm. Math. Phys. 165, 311-427 (1994)

[Ch] Chern, S.S.: Complex manifolds. Chicago Univ. of Chicago 1955/56

[Cl] Clemens, C.H. Jr.: Picard-Lefschetz theorem for families of nonsingular algebraic varieties acquiring ordinary singularities. Trans. AMS 136, 93-108 (1969)

[CF] Crane, L., Frenkel, I.B.: Four-dimensional topological quantum field theory, Hopf categories and the canonical bases. J. Math. Phys. 35, 5136-5154 (1994)

[EF] Etingof, P.I., Frenkel, I.B.: Central extensions of current groups in two dimensions. Commun. Math. Phys 165, 429-444 (1994)

[EK] Etingof, P.I., Khesin, B.A.: Affine Gelfand-Dickey brackets and holomorphic vector bundles. Geom. Funct. Anal. 4, 399-423 (1994)

[GS] Griffiths, P., Schmid, W.: Recent developments in Hodge theory: A discussion of techniques and results. In: Discrete subgroups of Lie groups and applications to moduli, Bombay Colloquium 1973, Oxford: Oxford Univ. Press, 1975, pp. 31-127

[L] Leray, J.: La calcul différential et intégral sur une variété analytique complexe (Problém de Cauchy, III) Bull. Soc. Math. France 87, 81-180 (1959)

[LMNS] Losev, A., Moore, G., Nekrasov, N., Shatashvili, S.: 4D avatars of 2D CFT, Talk at Annual USC meeting, March 1995

[M] Mickelsson, J.: Kac-Moody groups, topology of the Dirac determinant bundle and fermionization. Commun. Math. Phys. 110, 173-183 (1986)

[NS] Nair, V.P., Schiff, J.: Kähler-Chern-Simons theory and symmetries of anti-self-dual gauge fields. Nucl. Phys. B 371, 329-352 (1992)

[PS] Pressley, A., Segal, G.: Loop groups, Oxford: Clarendon Press, Oxford 1986

[T] Todorov, A.N.: Finiteness conditions for monodromy of families of curves and surfaces. Math. USSR Izvestia 10, 749-762 (1976)

[W1] Witten, E.: Non-abelian bozonization in two dimensions. Commun. Math. Phys. 92, 455472 (1984) 
[W2] Witten, E.: Quantum field theory and the Jones polynomial. Comm. Math. Phys. 121, 351 (1989)

[W3] Witten, E.: Chern-Simons gauge theory as a string theory. IASSNS-HEP 92/45, hepth/ 9207094

Communicated by G. Felder

Note added in proof. Since we submitted this paper there appeared two preprints related to our work:

[1] Losev, A., Moore, G., Nekrasov, N., Shatashvili, S.: Four-dimensional avatars of twodimensional RCFT, hep-th/9509151

[2] Losev, A., Moore, G., Nekrasov, N., Shatashvili, S.: Central extensions of gauge groups revisited, hep-th/9511185

The first paper extends the ideas of [LMNS]. In particular, the authors study the algebro-geometric sector of their theory and shed some light on the connection between our construction of the central extension $\hat{G}^{\Sigma}$ and their four-dimensional generalization of WZNW Lagrangean theory. In the second note the authors present the quotient realization for the gauge groups on an arbitrary manifold $M$ by elaborating the ideas of [PS, M]. Their construction has a purely real flavor, and it is based on ingenious introducing the currents on the cylinder over $M$. We note that the realization described in our paper also admits a generalization to current groups on a complex manifold of arbitrary dimension by using the $C^{\infty}$-version of Leray theory. However, the meromorphic setting exists only in the case of a Riemann surface $\Sigma$, and it makes the construction genuinely complex and rather rigid. 
